\title{
Gene-hunt gain for mental health
}

\author{
Flood of genetic locations linked to schizophrenia help spark financial boost to research field.
}

\section{BY SARA REARDON}

$\mathrm{R}$ esearchers seeking to unpick the complex genetic basis of mental disorders such as schizophrenia have taken a huge step towards their goal. A paper ${ }^{1}$ published in Nature this week ties 108 genetic locations to schizophrenia - most for the first time. The encouraging results come on the same day as a US\$650-million donation to expand research into psychiatric conditions.

Philanthropist Ted Stanley gave the money to the Stanley Center for Psychiatric Research at the Broad Institute in Cambridge, Massachusetts. The institute describes the gift as the largest-ever donation for psychiatric research.

"The assurance of a very long life of the centre allows us to take on ambitious long-term projects and intellectual risks," says its director, Steven Hyman.

The centre will use the money to fund genetic studies as well as investigations into the biological pathways involved in conditions such as schizophrenia, autism and bipolar disorder. The research effort will also seek better animal and cell models for mental disorders, and will investigate chemicals that might be developed into drugs.

The Nature paper $^{1}$ was produced by the Psychiatric Genomics Consortium (PGC) - a collaboration of more than 80 institutions, including the Broad Institute. Hundreds of researchers from the PGC pooled samples from more than 150,000 people, of whom 36,989 had been diagnosed with schizophrenia. This enormous sample size enabled them to spot 108 genetic locations, or loci, where the DNA sequence in people with schizophrenia tends to differ from the sequence in people without the disease. "This paper is in some ways proof that genomics can succeed," Hyman says.

"This is a pretty exciting moment in the history of this field," agrees Thomas Insel, director of the National Institute of Mental Health (NIMH) in Bethesda, Maryland, who was not involved in the study.

Many of the variations seem to be common, so most people will have some of them - but people with schizophrenia have more, and each contributes a small amount to the overall risk of developing the condition. This has made the variants difficult to spot in smaller samples, which is why the PGC collaboration is so crucial, Insel says: "If you want to look for common variants, you have to work with a lot of friends."

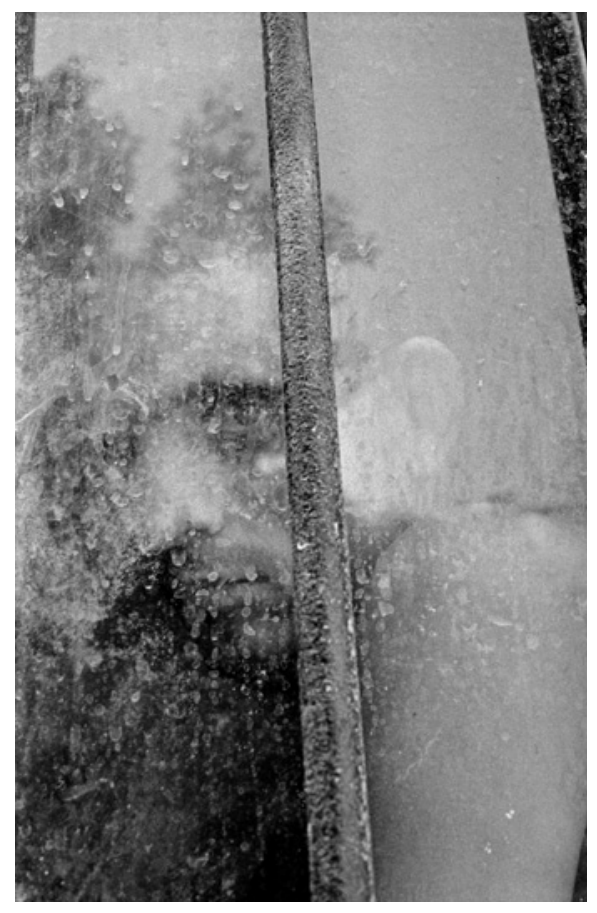

Broad population studies are shedding light on the genetic causes of mental disorders.

The large sample size also allowed the researchers to develop an algorithm that would calculate a 'risk score' for each variant's contribution to schizophrenia. This could eventually be used to predict who might develop the disorder or to add weight to an uncertain diagnosis of schizophrenia, Insel says. The NIMH is likely to dedicate more money soon towards doing intensive genetic surveys and following the genetic leads that come out of them, he adds.

Of the 108 loci identified in the PGC paper, 83 had not been identified by previous work. Many of the loci are in or near genes suspected to be involved in schizophrenia and other mental disorders, says Michael O'Donovan, a psychiatrist at Cardiff University, UK, who led the study. One was the gene encoding the dopamine receptor DRD2, which is a target of all current drugs for treating schizophrenia but has never come up as a risk factor. The loci also included genes that encode several proteins involved in transmitting electrical signals between neurons and creating connections between brain cells.

Several loci associated with schizophrenia encode proteins involved in the immune system, which has long been suspected to have a role in triggering the disorder. O'Donovan says that although the link is intriguing, its importance is not yet clear.

"It's a stunning confirmation that there are many, many genetic factors" involved in schizophrenia, says Pamela Sklar, a psychiatric geneticist at Mount Sinai School of Medicine in New York and a co-author of the paper. "There were hints of that from previous work, but this takes it to the next level." O'Donovan says that the PGC plans to double the sample size of people with schizophrenia by next year, which should enable the researchers to pinpoint the most important variants.

The group also plans to expand the data to include DNA from people around the world. The current collection, of nearly 200,000 samples, represents mainly people of northern European descent. "We want to make sure there is global health equity and that we don't develop treatments for just a subset of the world's population," says Hyman.

Schizophrenia is only one of the disorders that the group is working on. A 20 July paper in Nature Genetics ${ }^{2}$, with many of the same authors as the schizophrenia work, surveyed the genomes of 466 people with autism and some 2,500 people without, and estimated that about $52 \%$ of the risk for autism is genetic. Only $2.6 \%$ of the risk was linked to mutations that occur spontaneously during development, rather than being inherited. Like schizophrenia, autism seems to result largely from a certain combination of common variants, but people with autism are more likely also to have inherited a smaller number of rare variants that each greatly increase risk, the authors suggest. And because these variants are so rare, a larger sample size will be necessary to determine the truly important ones, says Joseph Buxbaum, a molecular biologist at Mount Sinai, who led the study.

The PGC is also using a platform called PsychChip, which targets parts of the genome associated with several psychiatric illnesses, including some less-studied ones such as anorexia nervosa and obsessive-compulsive disorder. By the end of this year, Sklar says, the team hopes to have sequenced samples from 100,000 people with mental illness: enough to identify the most meaningful associations. $=$ SEENEWS \& VIEWS P.412

1. Ripke, S. et al. Nature http://dx.doi.org/10.1038/ nature13595 (2014).

2. Gaugler, T. et al. Nature Genet. http://dx.doi. org/10.1038/ng.3039 (2014). 